\title{
Transatlantica
}

Revue d'études américaines. American Studies Journal

1 | 2017

Morphing Bodies: Strategies of Embodiment in Contemporary US Cultural Practices

\section{Aaron Shkuda, The Lofts of SoHo. Gentrification, Art and Industry in New York, 1950-1980}

\section{Pauline Chevalier}

\section{(2) OpenEdition}

\section{Journals}

Édition électronique

URL : https://journals.openedition.org/transatlantica/9234

DOI : $10.4000 /$ transatlantica.9234

ISSN : 1765-2766

Éditeur

Association française d'Etudes Américaines (AFEA)

Référence électronique

Pauline Chevalier, «Aaron Shkuda, The Lofts of SoHo. Gentrification, Art and Industry in New York, 1950-1980», Transatlantica [En ligne], 1 | 2017, mis en ligne le 16 octobre 2018, consulté le 24 mai 2021. URL : http://journals.openedition.org/transatlantica/9234 ; DOI : https://doi.org/10.4000/ transatlantica.9234

Ce document a été généré automatiquement le 24 mai 2021.

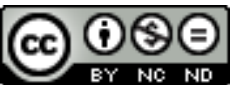

Transatlantica - Revue d'études américaines est mise à disposition selon les termes de la licence Creative Commons Attribution - Pas d'Utilisation Commerciale - Pas de Modification 4.0 International. 


\title{
Aaron Shkuda, The Lofts of SoHo. Gentrification, Art and Industry in New York, 1950-1980
}

\author{
Pauline Chevalier
}

\section{RÉFÉRENCE}

Aaron Shkuda, The Lofts of SoHo. Gentrification, Art and Industry in New York, 1950-1980, Chicago and London, The University of Chicago Press, 2016, ISBN-13: 978-0226334189, 320 pages, $45 \$$

1 «There are many reasons to celebrate the South Bronx, but some Bronx residents don't think a new artisanal coffee shop is one of them ${ }^{1}$. Alors que le New York Times avait placé le sud du Bronx comme un des nouveaux quartiers en vogue, les réactions furent vives, notamment de la part de nombreux artistes installés depuis parfois plusieurs décennies dans une zone en proie à la gentrification. La presse était attaquée pour sa complicité avec les promoteurs et son absence de recul critique face à un processus commun à toutes les grandes métropoles.

2 En novembre 2017, c'est un groupe d'activistes qui envahit l'exposition Laura Owens au Whitney Museum afin de dénoncer le rôle de l'artiste et de son galeriste Gavin Brown ${ }^{2}$ dans la gentrification de quartiers populaires de New York et de Los Angeles. Le slogan entonné dans les salles d'exposition est clair : «Laura Owens, Gavin Brown, our hoods unite to take you down! Leave our hoods and do what's right: Give your keys to Boyle Heights! $»^{3}$. Les membres de la "Boyle Heights Alliance Against Artwashing and Displacement » venus de Los Angeles s'étaient associés à d'autres collectifs, "The Brooklyn Anti-gentrification Network», «Equality for Flatbush», "Take Back the Bronx » ou encore « Decolonize This Place ». Autant d'associations, parfois fondées par des artistes, qui militent pour une prise de conscience par les artistes et les galeristes 
de leur rôle majeur dans le déplacement et la fragilisation des minorités et des classes populaires.

3 Le phénomène est loin d'être nouveau et le terme même de "gentrification ", forgé par Ruth Glass ${ }^{4}$ dans les années 1960, désigne un processus bien antérieur à sa qualification. Ce sont pourtant ces années 1960 qui figurent comme une référence pour comprendre les différents modèles de gentrification observables dans les métropoles actuelles. L'ouvrage d'Aaron Shkuda propose une étude très précise et fine de l'histoire de SoHo, quartier de New York dont l'évolution et la gentrification ont même donné lieu à une expression : la « SoHo-ization", pour désigner la récurrence du processus dans le reste de la métropole new-yorkaise et au-delà. Le phénomène, qui a vu un quartier industriel transformé par l'installation de nombreux artistes, de leurs ateliers, puis de galeries et de commerces luxueux, est largement connu.

4 Pourtant, aucune étude universitaire n'avait été menée de cette manière pour comprendre le rôle des artistes dans les processus de gentrification. Docteur de l'université de Chicago en histoire urbaine, Aaron Shkuda travaille depuis plus d'une dizaine d'années sur l'histoire de SoHo en adoptant une vision pluridisciplinaire. Son objet de recherche l'invitait en effet à mêler méthodes et disciplines pour saisir un phénomène qui a non seulement trait aux politiques de renouvellement urbain, mais aussi aux pratiques artistiques, à l'évolution du marché de l'art et à une histoire de l'architecture. Les travaux précédents sur le sujet proposaient souvent des points de vue plus circonscrits. Charles R. Simpson en 1981, dans SoHo: The Artist in the City ${ }^{5}$, étudie plus particulièrement l'installation illégale des artistes dans les anciens quartiers industriels et la construction d'une communauté soudée autour d'un quartier. L'ouvrage historique de Sharon Zukin, Loft Living, paru en 1982, se concentrait principalement sur le marché immobilier du loft et sur la naissance d'un mode de vie mis en place par les artistes. Si l'ouvrage proposait de riches développements sur les enjeux artistiques et esthétiques de la période, il accordait également une part importante aux analyses statistiques, économiques et démographiques. Richard Kostelanetz en 2003, dans The Rise and Fall of an Artists' Colony ${ }^{7}$, documente quant à lui la communauté d'artistes installés dans ce qui allait devenir SoHo. Son récit des années 1960 et 1970 est aussi celui d'un acteur de cette scène new-yorkaise. Plus récemment, Roslyn et Shael Shapiro revenaient sur l'histoire du 80 Wooster Street $^{8}$, immeuble historique au cœur de SoHo, acheté par George Maciunas, artiste Fluxus qui fut parfois désigné comme « le père de SoHo ». Cette étude, fréquemment citée par Aaron Shkuda, permettait aussi de relire l'histoire du quartier en se concentrant sur un immeuble bien particulier, et essentiel pour l'avant-garde new-yorkaise des années 1960 et 1970.

5 Alors que la gentrification devient une question majeure pour les artistes d'aujourd'hui, il manquait donc une étude pluridisciplinaire sur ce qui est devenu un modèle historique, SoHo. Aaron Shkuda s'intéresse en effet à l'idée même du modèle: le quartier de SoHo propose un schéma d'évolution qui semble avoir été repris dans de nombreux quartiers. L'auteur insiste cependant sur la spécificité de SoHo et sur la notion même de modèle. Si cette évolution a été littéralement et consciemment reprise par certains promoteurs immobiliers et par plusieurs municipalités, comme une influence directe, SoHo constitue surtout un paradigme, un outil de l'analyse des processus de gentrification et de renouvellement urbain. L'auteur se réfère régulièrement, au fil de son discours, à d'autres expériences : Chicago, San Francisco ou encore Berlin, afin de sans cesse replacer le cas new-yorkais dans un contexte plus 
large et ainsi valider son postulat de départ : l'histoire de SoHo permet de construire une grille de repères pour l'étude de la transformation des quartiers industriels depuis la fin des années 1950.

6 L'ouvrage repose sur la singularité de son sujet qui lui permet de se distinguer parmi les travaux historiques sur la gentrification cités en introduction: les artistes qui se sont installés dans SoHo ont mis en place un processus alternatif de renouvellement urbain. «By renovating industrial lofts that policy makers viewed as slums, artists produced a new use for underutilized industrial space. In creating the residential loft and lobbying for its regularization, SoHo artist groups posited a new postindustrial future for New York City that did not rely on slum clearance or urban renewal (...) during a time of economic decline in New York, SoHo artists provided a way to generate residential and commercial real estate investment in industrial areas without the economic and social costs of slum »(8-11). C'est donc à cette spécificité de la transformation urbaine par les artistes, sans destruction, que s'intéresse l'auteur en saisissant l'influence du processus sur le phénomène de gentrification, une gentrification «menée par les artistes » (« artist-led gentrification »), souvent de façon non intentionnelle, quand beaucoup d'entre eux en furent les premières victimes.

7 L'auteur organise son propos de façon chronologique, en faisant d'abord le récit des lieux, dès le XIX ${ }^{e}$ siècle, au temps de l'industrie florissante. Les immeubles à la structure en fonte («cast iron »), inspirés de palais italiens, construits à moindre coût grâce à cette nouvelle technique, accueillaient de nombreuses petites industries. L'auteur suit l'évolution d'une entreprise, Weiss \& Klau, depuis la fin du XIX' siècle, jusqu'en 1966 et l'effondrement des sols de l'immeuble. L'auteur ponctue son récit d'anecdotes historiques, grâce à de nombreuses sources tirées de la presse de l'époque. On y croise par exemple Elisha Grave Otis, inventeur en 1853 du frein de sécurité des ascenseurs qui furent alors développés dans ces nouveaux immeubles au sud de Manhattan. Cette enquête historique est régulièrement rapportée à la période qui constitue le cœur de l'ouvrage : les années 1960 et 1970 . Quelle industrie occupait l'immeuble bien avant que Paula Cooper y installe la première galerie du quartier en 1968 ? Que trouvait-on à la place du restaurant Food au XIX ${ }^{\mathrm{e}}$ siècle ? Ces références ne sont pas qu'anecdotiques, elles permettent de témoigner de la spécificité de SoHo, contre les comparaisons trop rapides entre différents processus de renouvellement urbain. En outre, l'auteur montre comment la persistance de la petite industrie locale jusque dans les années 1960 a permis de sauver le quartier de la destruction, avant que les artistes ne prennent le relais.

8 C'est à partir de cette particularité industrielle que les artistes ont pu s'installer dans SoHo avant même que cette appellation n'existe. L'architecture - luminosité et vastes espaces - était aussi propice, au même titre que le prix dérisoire des loyers. L'habitation était cependant illégale. S'il était possible d'y installer son atelier, la résidence n'était pas encore autorisée dans ces quartiers destinés à l'industrie. L'auteur cite également la proximité de Greenwich Village dans l'attraction exercée par SoHo, mais il n'aborde pas la convergence entre ces nouveaux territoires et les nouvelles pratiques artistiques qui appelaient l'usage de matériaux de récupération, de détritus qui seront ensuite trouvés dans les rues de SoHo.

9 L'histoire du quartier retracée par l'auteur est relativement bien connue, on la rencontrait chez Simpson et Zukin dans leurs ouvrages publiés au début des années 1980. L'auteur apporte cependant de nouvelles sources et, par de nombreux entretiens 
avec des artistes, des acteurs de cette période, il nourrit une historiographie encore lacunaire. Il tente notamment de saisir les parcours des artistes qui s'installent dans SoHo, qui ont souvent une formation universitaire, dont les familles pouvaient parfois subvenir à leurs besoins, mais qui éprouvaient quelques difficultés à gagner leur vie indépendamment. Ces informations aident aussi à comprendre les enjeux de la préservation du quartier pour les artistes ainsi que la récurrence du modèle. Elles auraient pu être plus détaillées encore. L'homogénéité de la communauté d'artistes de SoHo, artistes blancs, ayant suivi des études supérieures, issus de classes moyennes aisées, correspond à un aspect essentiel du processus de gentrification étudié par l'auteur.

De même, alors qu'Aaron Shkuda développe quelques paragraphes sur le rôle de George Maciunas, il omet de remarquer que les lofts étaient répartis bien au-delà de SoHo avant l'achat de plusieurs immeubles par l'artiste qui va donner une impulsion considérable à l'installation des artistes dans SoHo. On peut regretter l'absence d'analyse de la répartition des lofts. S'il est souvent admis que la communauté d'artistes a investi SoHo, les archives ${ }^{9}$ témoignent d'une installation beaucoup plus éparse. La lente légalisation de certains lofts par la mise en place du programme «Artist in Residence » dès 1962 n'incite pas les artistes à se regrouper dans SoHo. Les listes d' "A.I.R. » montrent au contraire que les lofts sont répartis dans Midtown, dans Chelsea, dans un périmètre qui dépasse très largement les limites de SoHo. Ce n'est qu'à partir des projets de coopératives de George Maciunas - des achats groupés de lofts et d'immeubles, que la communauté va se resserrer autour de quelques «blocks » précis au sud de Houston Street.

11 En consultant la presse, House and Garden, Life magazine, l'auteur montre comment s'est peu à peu répandue l'esthétique industrielle du loft sur les pages de papier glacé. Ce rôle de la presse dans la visibilité de la communauté d'artistes, dans la diffusion de l'esthétique du loft et dans la promotion des nouveaux quartiers réhabilités est sans cesse mis en valeur par l'auteur qui, de cette manière, révèle peu à peu combien le processus de gentrification a échappé aux artistes eux-mêmes. C'est là que l'auteur parvient à montrer la spécificité de SoHo et les limites du modèle comme influence (et non comme outil d'analyse). L'ouvrage permet en effet de saisir le paradoxe d'un quartier défendu par des artistes, contre le renouvellement urbain qui impliquait alors la destruction d'un patrimoine architectural non reconnu comme tel, notamment avec le projet de Lower Manhattan Expressway, mais qui ne put accueillir la communauté d'artistes que relativement brièvement.

12 Si les associations de défense du quartier et de la communauté d'artistes, telles que l' "Artist Tenants Association ", puis la "SoHo Artists Association» ont permis de progressivement légaliser la résidence des artistes, elles ont aussi ouvert la voie aux promoteurs immobiliers. La modification des «zones» dans le plan d'urbanisme est alors cruciale et l'est toujours aujourd'hui. Lorsqu'un quartier passe d'une zone réservée aux petites industries, avec des dérogations pour les artistes, à une zone déclarée comme mixte ou résidentielle, les immeubles peuvent être transformés en habitation dont la rentabilité augmente de façon radicale. Ce processus est toujours celui qui aujourd'hui conduit à l'éviction d'artistes dans les quartiers du canal de Gowanus par exemple, ou encore à Greenpoint ou Red Hook.

Cette difficulté et cet équilibre précaire dans la légalisation des lofts sont au cœur de la définition de la "SoHo-ization »: "Artists' ceaseless efforts to change laws to secure 
legal recognition of their right to turn deserted industrial lofts into their living as well as work spaces played a key, if initially unanticipated, role in pioneering new strategies for urban renewal. Yet, carrying this argument forward, it is not hard to see where conflicts could emerge. If artists' actions resulted in eliminating slums and upgrading neighborhoods, they were also helping to increase the value of local property. If this pattern continued, it would not be long before this increase in the cost of the area lofts became an issue, both for artists and the industries with which they shared space " (105). Les artistes prennent directement conscience de leur rôle dans la transformation de ces quartiers industriels et tentent de préserver l'activité des manufactures qui rythme encore les lieux. Pourtant, l'arrivée des galeries, celles de Paula Cooper, rencontrée par l'auteur et dont le récit éclaire bien le propos, va creuser le sillon qui permettra l'arrivée en masse d'une classe aisée, attirée par ces nouveaux territoires du marché de l'art. L'appellation «SoHo » est alors directement liée à la reconfiguration des galeries dans Manhattan et à leur valorisation.

Les galeries, les festivals organisés par les artistes et la presse admirative de ces nouveaux modes de vie, attiraient de plus en plus de visiteurs qui pouvaient désormais s'installer dans le quartier. Le «loft » perdait de sa singularité : conçu comme une configuration idéale entre espace de travail et lieu de vie, il devenait le type d'habitat que le marché immobilier recherchait pour transformer ces quartiers industriels sauvés de la destruction et dont on commençait à admirer le patrimoine architectural. L'architecture intérieure prône une esthétique industrielle relayée dès 1979 par un magasin comme Macy's (179). Les artistes qui avaient développé cette esthétique par commodité, en installant par exemple des fours ou des éviers industriels achetés dans les manufactures du quartier, se voyaient désignés comme les pionniers d'un nouvel habitat. L'ouvrage détaille alors avec une grande précision la concomitance du déclin industriel et de la croissance des investissements immobiliers dans le quartier.

SoHo, zone industrielle, présente cependant quelques caractéristiques qui ne sont pas toujours celles des quartiers en proie à la gentrification aujourd'hui : "Although the neighborhood did not house lower-income residents of color, loft investment in SoHo threatened to dislocate a relatively low-income group, artists as well as industrial businesses, which employed African American and Latino workers » (207). De même, les premiers investissements immobiliers ne provenaient pas de grandes firmes qui arrivèrent plus tard et qui œuvrent aujourd'hui en premier dans les nouveaux quartiers en mutation. La législation mise en place par la municipalité de New York permit ensuite de confirmer un nouveau mode de renouvellement urbain par la transformation des immeubles industriels en lofts résidentiels. Un processus inventé par les artistes qui en avaient, pour une partie d'entre eux, perdu le contrôle.

16 Alors que le début des années 1980 voit croître les références à SoHo dans d'autres métropoles nord-américaines, aux États-Unis et au Canada, le terme "gentrification " commence à être utilisé pour SoHo. En faisant le récit du quartier pour qui parler de "gentrification » au début des années 1960 serait anachronique, l'auteur donne à voir toute la complexité du phénomène à partir d'un exemple historique dont la reprise a parfois balayé la spécificité. Même si l'auteur ne l'affirme pas ainsi, son ouvrage propose non seulement une enquête historique remarquable, grâce à l'abondance et à la variété des sources utilisées, mais constitue également un véritable manuel de compréhension, voire de lutte, contre les processus de gentrification, pour contourner 
un mécanisme désormais bien rôdé qui transforme de nombreux artistes en pionniers du renouvellement urbain et d'une injustice sociale qui ne dit pas toujours son nom.

\section{NOTES}

1. Sandra E. Garcia, "Strong Reactions in the South Bronx After It's Called a 'Place to Go' ", The New York Times, February 8, 2017.

2. Le galeriste qui a ouvert une galerie dans Harlem et une autre dans Chinatown à New York est aussi l'initiateur d'un projet, avec Laura Owens, dans le quartier de Boyle Heights à Los Angeles, « 356 Mission », qui depuis 2013 opère comme un espace d'exposition et de performance engagé dans la vie du quartier.

3. Benjamin Sutton, «Anti-gentrification Activits Protest Laura Owens Exhibition at the Whitney Museum », Hyperallergic, November 10, 2017 (https://hyperallergic.com/411134/antigentrification-activists-protest-laura-owens-exhibition-at-the-whitney-museum/)

4. Ruth Glass, London: Aspects of Change, Londres, MacGibbon \& Kee, 1964.

5. Charles R. Simpson, SoHo : the Artist in the City, Chicago, The University of Chicago Press, 1981.

6. Sharon Zukin, Loft Living: Culture and Capital in Urban Change, Baltimore and London, The Johns Hopkins University Press, 1982.

7. Richard Kostelanetz, SoHo : the Rise and Fall of an Artist's Colony, New York, Routledge, 1983.

8. Roslyn et Shael Shapiro, Illegal Living : 80 Wooster Street and the Evolution of SoHo, New York, Jonas Mekas Foundation, 2010.

9. Artist Tenants Association Records, 1959-1978, Archives of American Art, Smithsonian Institution, Washington D.C.

INDEX

Thèmes : Recensions

\section{AUTEURS}

PAULINE CHEVALIER

Université de Franche-Comté 